PROCEEDINGS OF THE

AMERICAN MATHEMATICAL SOCIETY

Volume 127, Number 7, Pages 2019-2028

S 0002-9939(99)05062-5

Article electronically published on February 26, 1999

\title{
HOMOGENIZATION OF THE INVISCID INCOMPRESSIBLE FLUID FLOW THROUGH A 2D POROUS MEDIUM
}

\author{
ANDRO MIKELIĆ AND LAETITIA PAOLI \\ (Communicated by James Glimm)
}

\begin{abstract}
We consider the non-stationary incompressible Euler equations in a $2 \mathrm{D}$ porous medium. We suppose a periodic porous medium, with the period proportional to the characteristic pore size $\varepsilon$ and with connected fluid part. The flow is subject to an external force, corresponding to an inflow. We start from an initial irrotational velocity and prove that the effective filtration velocity satisfies a transient filtration law. It has similarities with Darcy's law, but it now connects the time derivative of the filtration velocity with the pressure gradient. The viscosity does not appear in the filtration law any more and the permeability tensor is determined through auxiliary problems of decomposition type. Using the limit problem, we construct the correction for the fluid velocity and prove that $C^{1}\left([0, T] ; L^{2}(\Omega)^{2}\right)$-norm of the error is of order $\varepsilon$. Similarly, we estimate the difference between the fluid pressure and its correction in $C\left([0, T] ; L^{1}(\Omega)\right)$ as $C \varepsilon$.
\end{abstract}

\section{INTRODUCTION}

The derivation of filtration laws in a porous medium by homogenization of incompressible Navier-Stokes equations is nowadays well understood. Starting from Tartar's rigorous justification of Darcy's law by homogenization of the Stokes system in a periodic porous medium (see e.g. the review paper of G. Allaire [1]), this method allowed also to treat the inertia effects (see A. Mikelić [8], A. Bourgeat, E. Marušić-Paloka, A. Mikelić [2]). However, having the Reynolds number of an order in $\varepsilon$ higher than the critical value determined in A. Mikelić [8], the asymptotic analysis of the Navier-Stokes system gives the stationary "two-pressures" Euler system with the Prandtl boundary layer at the local cell level and such systems do not seem to be well-posed.

On the other hand, it is important to consider underground flows of fluids with small viscosity. Having in mind the crucial role played by the viscosity in the derivation of Darcy's law, it is questionable whether the linear relation between the filtration velocity and the pressure gradient can be still used. This question can be clarified only by considering the upscaling of the non-stationary incompressible Euler system in a porous medium, with a correct choice of the dimensionless characteristic numbers.

Underground flows are governed by inflows and outflows, or by pressure drops at the boundary. However, the simultaneous study of boundary layers and the flow

Received by the editors September 15, 1997.

1991 Mathematics Subject Classification. Primary 35B27, 76C05, 76S05.

(C)1999 American Mathematical Society 
equations lead to significant technical complications (see e.g. [7]). In view of it, we introduce instead an external force caused by the boundary data and consider the incompressible Euler system in 2D. At the outer boundary we impose periodic boundary conditions.

The dimensionless form of the equation reads as follows:

$$
\frac{L}{T U} \frac{\partial u^{\varepsilon}}{\partial t}+\left(u^{\varepsilon} \nabla\right) u^{\varepsilon}+\nabla p^{\varepsilon}=F^{\varepsilon} \frac{L}{U^{2}},
$$

where $L$ is the characteristic length, $U$ is the characteristic velocity and $T$ is the characteristic time. The equation (1.1) is valid in the fluid part of the domain and $L=O(1)$. Then the transient flow regime is described by the choice $T=O(1)$. Moreover, $F^{\varepsilon}$ is the body force and $F^{\varepsilon}=O(\varepsilon)$.

The choice of the characteristic velocity determines completely the effective filtration. The formal asymptotic expansion leads to the following three possibilities:

- if $U$ is of order $o(\varepsilon)$, then the filtration law involves a linear relationship between the time derivative of the velocity and the pressure gradient,

- if $U$ is of order bigger than $O(\varepsilon)$, we meet the same type of difficulties as in the case of the Navier-Stokes system with the high Reynolds number and it is a kind of turbulent filtration,

- finally, if the characteristic velocity behaves as $O(\varepsilon)$, the terms in the material derivative of the velocity are of the same order at the pore level and the inertial term contributes to the approximation of the pressure.

Consequently, the case $U=O(\varepsilon)$ is the most interesting non-turbulent filtration regime leading to a new constutive relation which does not involve viscosity. Its practical importance is limited only by the fact that, in realistic situations, such flow is usually unsaturated and the presence of air slows the flow. Then $F^{\varepsilon}=\varepsilon f$ guarantees that $u^{\varepsilon}=O(1)$ and the underlying initial value problem is:

$$
\begin{gathered}
\frac{\partial u^{\varepsilon}}{\partial t}+\varepsilon\left(u^{\varepsilon} \nabla\right) u^{\varepsilon}+\varepsilon \nabla p^{\varepsilon}=f(x, t) \quad \text { in } \Omega^{\varepsilon} \times(0, T), \\
\operatorname{div}\left(u^{\varepsilon}\right)=0 \quad \text { in } \Omega^{\varepsilon} \times(0, T), \\
u^{\varepsilon} . \nu=0 \quad \text { on }\left(\partial \Omega^{\varepsilon} \backslash \partial \Omega\right) \times(0, T), \quad\left\{u^{\varepsilon}, p^{\varepsilon}\right\} \text { is } \Omega \text {-periodic, } \\
u^{\varepsilon}(x, 0)=u_{0}^{\varepsilon}(x)=u_{0}\left(x, \frac{x}{\varepsilon}\right) \quad \text { in } \Omega^{\varepsilon},
\end{gathered}
$$

where $\Omega^{\varepsilon}$ is the fluid part of the domain $\Omega$.

For simplicity, we suppose that the porous medium $\Omega$ is a rectangle $\Omega=(0, L)^{2}$ and the corresponding space-time cylinder $\Omega \times(0, T)$ is denoted $Q_{T}$. With $\Omega$ we associate a microstructure through the periodic cell $Y=(0,1)^{2}$, made of two complementary parts: the solid part $\mathcal{O}, \overline{\mathcal{O}} \subset \subset Y$, and the fluid part $\mathcal{Y}=Y \backslash \overline{\mathcal{O}}$. We suppose that $S=\partial \mathcal{O} \in C^{\infty}, \mathcal{Y}$ is connected and $\Omega=\bigcup_{k} \varepsilon(k+Y)$, i.e that $\Omega=(0, L)^{2}$ is covered by a regular mesh of size $\varepsilon$ (the pore size). Finally, we denote $\Omega^{\varepsilon}=\bigcup_{k} \varepsilon(\mathcal{Y}+k)$ the fluid part of $\Omega$.

The main results of this paper are twofold: Firstly, we are going to homogenize the Euler system (1.2)-(1.5) containing inertia effects at the pore level, which is the first result of this type in the literature. Secondly, we will obtain a new class of transient filtration laws which do not involve viscosity. Moreover, we are going to prove the convergence of the homogenization process through an $L^{2}$ estimate of order $O(\varepsilon)$ for the velocity and of order $O(1)$ not for the pressure itself, but for a pseudo-pressure taking into account the velocity. 


\section{DERIVATION OF THE TRANSIENT FILTRATION LAW}

We suppose $f \in C_{\text {per }}^{\infty}\left(Q_{T}\right)^{2}$ and $u_{0} \in \mathcal{H}_{\text {per }}^{\infty}$ with $\mathcal{H}_{\text {per }}^{\infty}=\left\{v \in C_{\text {per }}^{\infty}(\Omega \times \mathcal{Y})^{2}\right.$ : $\operatorname{div}_{x} v(x, y)=\operatorname{div}_{y} v(x, y)=0$ in $\Omega \times \mathcal{Y}, v \cdot \nu=0$ on $\left.\Omega \times S\right\}$. Then the classical theory (see e.g. P.L. Lions [5] or C. Marchioro, M. Pulvirenti [6]) implies the existence of a unique solution

$$
\left\{u^{\varepsilon}, p^{\varepsilon}\right\} \in C^{1}\left([0, T] ; C^{\infty}\left(\overline{\Omega^{\varepsilon}}\right)^{2}\right) \times C\left([0, T] ; C^{\infty}\left(\overline{\Omega^{\varepsilon}}\right) \cap L^{2}\left(\Omega^{\varepsilon}\right)\right)
$$

for the problem (1.2)-(1.5), satisfying $\int_{\Omega^{\varepsilon}}\left(p^{\varepsilon}+\frac{1}{2}\left|u^{\varepsilon}\right|^{2}\right)=0$ for every $t \in[0, T]$.

Let us recall the Lamb formula in $2 \mathrm{D}$ :

$$
(u \nabla) u=\nabla\left(\frac{1}{2}|u|^{2}\right)-u \wedge \omega,
$$

where $\omega=\operatorname{curl}(u)$. Then, denoting $\omega^{\varepsilon}=\operatorname{curl}\left(u^{\varepsilon}\right)$, we rewrite (1.2) as

$$
\frac{\partial u^{\varepsilon}}{\partial t}-\varepsilon u^{\varepsilon} \wedge \omega^{\varepsilon}+\varepsilon \nabla\left(\frac{1}{2}\left|u^{\varepsilon}\right|^{2}+p^{\varepsilon}\right)=f(x, t) \quad \text { in } \Omega^{\varepsilon} \times(0, T) \text {. }
$$

Taking the curl of this equation, we obtain that $\omega^{\varepsilon}$ is a smooth solution of the following transport equation:

$$
\frac{\partial \omega^{\varepsilon}}{\partial t}+\varepsilon u^{\varepsilon} \nabla \omega^{\varepsilon}=\operatorname{curl} f \quad \text { in } \Omega^{\varepsilon} \times(0, T) .
$$

Hence, with the classical results of V.I. Judovič [4], we obtain an expression of $\omega^{\varepsilon}$ as

$$
\omega^{\varepsilon}(x, t)=\omega_{0}^{\varepsilon}\left(\Phi^{\varepsilon}(-t, x)\right)+\int_{0}^{t} \operatorname{curl} f\left(s, \Phi^{\varepsilon}\left(s, \Phi^{\varepsilon}(-t, x)\right)\right) d s,
$$

where $\Phi^{\varepsilon}$ denotes the flow associated with the ODE $\dot{y}=\varepsilon u^{\varepsilon}(t, y)$ and $\omega_{0}^{\varepsilon}=\operatorname{curl}\left(u_{0}^{\varepsilon}\right)$. Thus, we obtain the following a priori estimates for $\left\{u^{\varepsilon}, \omega^{\varepsilon}\right\}$ :

Proposition 2.1. Let $\omega_{0}^{\varepsilon}=\operatorname{curl}\left(u_{0}^{\varepsilon}\right)$ and $\omega^{\varepsilon}=\operatorname{curl}\left(u^{\varepsilon}\right)$. Then we have

$$
\begin{gathered}
\left\|\omega^{\varepsilon}\right\|_{L^{\infty}\left(\Omega^{\varepsilon} \times(0, T)\right.} \leq\left\|\omega_{0}^{\varepsilon}\right\|_{L^{\infty}\left(\Omega^{\varepsilon}\right)}+\int_{0}^{T}\|\operatorname{curl} f(t)\|_{L^{\infty}\left(\Omega^{\varepsilon}\right)} d t, \\
\left\{\begin{array}{c}
\left\|u^{\varepsilon}\right\|_{L^{\infty}\left(0, T ; L^{2}\left(\Omega^{\varepsilon}\right)^{2}\right)} \leq\left\|u_{0}^{\varepsilon}\right\|_{L^{2}\left(\Omega^{\varepsilon}\right)^{2}}+\int_{0}^{T}\|f(t)\|_{L^{2}\left(\Omega^{\varepsilon}\right)^{2}} d t, \\
\left\|\partial_{t} u^{\varepsilon}\right\|_{L^{\infty}\left(0, T ; L^{2}\left(\Omega^{\varepsilon}\right)^{2}\right)} \leq\|f\|_{L^{\infty}\left(0, T ; L^{2}\left(\Omega^{\varepsilon}\right)^{2}\right)} \\
+\varepsilon\left\|u^{\varepsilon}\right\|_{L^{\infty}\left(0, T ; L^{2}\left(\Omega^{\varepsilon}\right)^{2}\right)}\left\|\omega^{\varepsilon}\right\|_{L^{\infty}\left(\Omega^{\varepsilon} \times(0, T)\right) .}
\end{array}\right.
\end{gathered}
$$

Proof. Estimate (2.3) is immediate with (2.2) and (2.4) follows by taking successively $u^{\varepsilon}$ and $\partial_{t} u^{\varepsilon}$ as test-functions in (2.1).

From now on we suppose in addition $\operatorname{curl}_{y}\left(u_{0}\right)=0$ in $\Omega \times \mathcal{Y}$. Then the estimates (2.3)-(2.4) imply that $\omega^{\varepsilon}, u^{\varepsilon}$ and $\partial_{t} u^{\varepsilon}$ are uniformly bounded with respect to $\varepsilon$ in appropriate norms.

Remark 2.2. A cursory glance at the velocity estimate in V.I. Judovič [4] suffices to find out that (2.3)-(2.4) do not imply a uniform bound for $\nabla u^{\varepsilon}$, since the flow has non-zero circulations around solid inclusions. 
Therefore it is reasonable to try the following two-scale asymptotic expansion for $u^{\varepsilon}$ and $p^{\varepsilon}$ :

$$
\begin{gathered}
u^{\varepsilon}(x, t)=u^{0}\left(x, \frac{x}{\varepsilon}, t\right)+\varepsilon u^{1}\left(x, \frac{x}{\varepsilon}, t\right)+\ldots, \\
p^{\varepsilon}(x, t)=\frac{1}{\varepsilon} p^{0}(x, t)+p^{1}\left(x, \frac{x}{\varepsilon}, t\right)+\ldots
\end{gathered}
$$

After standard calculations, we obtain the following system of PDE's, coupling the microstructure and the macrostructure:

$$
\begin{gathered}
\frac{\partial u^{0}}{\partial t}+\left(u^{0} \nabla_{y}\right) u^{0}+\nabla_{y} p^{1}+\nabla_{x} p^{0}=f \quad \text { in } Q_{T} \times \mathcal{Y}, \\
\operatorname{div}_{y}\left(u^{0}\right)=0 \quad \text { in } Q_{T} \times \mathcal{Y}, \quad u^{0} . \nu=0 \quad \text { in } Q_{T} \times S, \\
\left\{u^{0}, p^{1}\right\} \text { is 1-periodic in } y, \quad u^{0}(x, y, 0)=u_{0}(x, y) \quad \text { in } \Omega \times \mathcal{Y}, \\
\operatorname{div}_{x}\left(\int_{\mathcal{Y}} u^{0}(x, y, t) d y\right)=0 \quad \text { in } Q_{T}, \quad\left\{u^{0}, p^{0}\right\} \text { is } \Omega \text {-periodic in } x .
\end{gathered}
$$

We transform (2.7) by using the Lamb formula and get

$$
\frac{\partial u^{0}}{\partial t}-u^{0} \wedge \omega^{0}+\nabla_{y}\left(p^{1}+\frac{1}{2}\left|u^{0}\right|^{2}\right)+\nabla_{x} p^{0}=f \quad \text { in } Q_{T} \times \mathcal{Y},
$$

with $\omega^{0}=\operatorname{curl}_{y}\left(u^{0}\right)$. Then, taking the curl of this equation, we obtain that the vorticity field satisfies a homogeneous linear transport equation with zero initial value (since we suppose $\left.\operatorname{curl}_{y}\left(u_{0}\right)=0\right)$ and has $(0, T) \times S$ as characteristic boundary $x$-(a.e.). Hence, for any Lipschitz function $u^{0}$, we have $\omega^{0}=0$ and (2.11) becomes

$$
\frac{\partial u^{0}}{\partial t}+\nabla_{y}\left(p^{1}+\frac{1}{2}\left|u^{0}\right|^{2}\right)+\nabla_{x} p^{0}=f \quad \text { in } Q_{T} \times \mathcal{Y} .
$$

Now the problem described by (2.8)-(2.10) and (2.12) can be analysed as a linear problem by defining a pseudo-pressure field $\tilde{\pi}^{0}$ by:

$$
\tilde{\pi}^{0}(x, y, t)=p^{1}(x, y, t)+\frac{1}{2}\left|u^{0}\right|^{2}(x, y, t) \quad \text { in } Q_{T} \times \mathcal{Y} .
$$

Thus, we are able to separate fast and slow scales by setting:

$$
u^{0}(x, y, t)=u_{0}(x, y)+K(y) \int_{0}^{t}\left(f(x, \tau)-\nabla_{x} p^{0}(x, \tau)\right) d \tau .
$$

The matrix $K$ is given by:

$$
K_{i j}(y)=w_{i}^{j}(y),
$$

and $\left\{w^{i}, \pi^{i}\right\}$ is the unique solution for the decomposition problem

$$
\left\{\begin{array}{l}
w^{i}+\nabla_{y} \pi^{i}=e_{i} \quad \text { in } \mathcal{Y}, \quad \operatorname{div}_{y}\left(w^{i}\right)=0 \quad \text { in } \mathcal{Y}, \\
w^{i} \cdot \nu=0 \quad \text { on } S, \quad\left\{w^{i}, \pi^{i}\right\} \quad \text { is 1-periodic in } y, \\
\int_{\mathcal{Y}} \pi^{i}=0,
\end{array}\right.
$$

where $e_{i}$ is the $i$-th canonical basis vector of $\mathbb{R}^{2}$. 
Now let $K_{E}=\int_{\mathcal{Y}} K(y) d y$ be the "Euler permeability" tensor. Obviously $K_{E}$ is symmetric and positive definite. The "Euler filtration velocity" is then $u_{E}(x, t)=$ $\int_{\mathcal{Y}} u^{0}(x, y, t) d y$ and the effective flow is described by the following equations:

$$
\left\{\begin{aligned}
& u_{E}(x, t)= \int_{\mathcal{Y}} u_{0}(x, y) d y+K_{E} \int_{0}^{t}\left(f(x, \tau)-\nabla_{x} p^{0}(x, \tau)\right) d \tau \\
& \text { in } Q_{T} \text { (Euler-Darcy's filtration law), } \\
& \operatorname{div}_{x}\left(u_{E}\right)=0 \quad \text { in } Q_{T}, \quad \int_{\Omega} p^{0}(x, t) d x=0 \quad \text { in }[0, T], \\
&\left\{u_{E}, p^{0}\right\} \text { is } L \text {-periodic. }
\end{aligned}\right.
$$

In the next section, we prove the existence of a unique smooth solution for the problem (2.7)-(2.10) satisfying

$$
\left\{\begin{array}{l}
\int_{\Omega} p^{0}(x, t) d x=0 \quad \text { in }[0, T] \\
\int_{\mathcal{Y}}\left(p^{1}(x, y, t)+\frac{1}{2}\left|u^{0}\right|^{2}(x, y, t)\right) d y=0 \quad \text { in } \Omega \times[0, T] .
\end{array}\right.
$$

Since (2.17) has a unique smooth solution, we have immediately the equivalence between the problem (2.7)-(2.10) with condition (2.18) and the problem (2.14)(2.17).

Then, in section 4 , we estimate the $L^{2}$-norm of the difference between $u^{\varepsilon}$ and $u^{0}\left(x, \frac{x}{\varepsilon}, t\right)$ as $C \varepsilon$, uniformly in time. Similarly the $C\left([0, T] ; L^{1}\left(\Omega^{\varepsilon}\right)\right)$-norm of the difference between $\varepsilon p^{\varepsilon}$ and $p^{0}$ is also estimated as $C \varepsilon$.

Finally, some Rayleigh-Maxwell type formulas for the permeability tensor are given.

\section{EXISTENCE AND UNiqueness For the two-PRESSURES EUler EQUATIONS}

The theory of the two-pressures Navier-Stokes system, analogous to (2.7)-(2.10), is quite complicated and it was only possible to prove existence and uniqueness for $f$ with small Lipschitz norm in the stationary case (see A. Mikelić [8] and E. Marušić-Paloka, A. Mikelić [7]). On the contrary, we prove that under the hypothesis $\operatorname{curl}_{y}\left(u_{0}\right)=0$, the 2D two-pressures Euler system with condition (2.18) has a unique smooth global solution.

We start with the existence of a smooth solution. Let $p^{0} \in C^{\infty}\left([0, T] ; C_{p e r}^{\infty}(\Omega)\right)$ be defined by:

$$
\left\{\begin{array}{l}
\operatorname{div}_{x}\left(K_{E} \int_{0}^{t}\left(f(x, \tau)-\nabla_{x} p^{0}(x, \tau)\right) d \tau\right)=0 \quad \text { in } \Omega \times[0, T], \\
\int_{\Omega} p^{0}(x, t) d x=0 \quad \text { in }[0, T] .
\end{array}\right.
$$

Now we set

$$
u^{0}(x, y, t)=u_{0}(x, y)+K(y) \int_{0}^{t}\left(f(x, \tau)-\nabla_{x} p^{0}(x, \tau)\right) d \tau,
$$




$$
\tilde{\pi}^{0}(x, y, t)=\sum_{k=1}^{2} \pi^{k}(y)\left(f_{k}(x, t)-\frac{\partial p^{0}}{\partial x_{k}}(x, t)\right) .
$$

Obviously, $\left\{u^{0}, \tilde{\pi}^{0}\right\} \in C^{\infty}(\bar{\Omega} \times \overline{\mathcal{Y}} \times[0, T])^{3}$ and we have the following result:

Proposition 3.1. Let the functions $p^{0}, u^{0}$ and $\tilde{\pi}^{0}$ be given by (3.1)-(3.3) and let $p^{1}=\tilde{\pi}^{0}-\frac{1}{2}\left|u^{0}\right|^{2}$. Then the triple $\left\{u^{0}, p^{1}, p^{0}\right\}$ is a solution for the problem (2.7)(2.10) with condition (2.18).

Therefore we have established the existence of at least one smooth solution for (2.7)-(2.10). It remains to prove the uniqueness. We have

Proposition 3.2. The triple $\left\{u^{0}, p^{1}, p^{0}\right\}$ is a unique smooth solution for the twopressures Euler equations (2.7)-2.10, satisfying condition (2.18).

Proof. Step 1. Let $\left\{u^{0}, p^{1}, p^{0}\right\}$ be a smooth solution for (2.7)-(2.10). Then $\omega^{0}=$ $\operatorname{curl}_{y}\left(u^{0}\right)$ satisfies:

$$
\left\{\begin{array}{l}
\frac{\partial \omega^{0}}{\partial t}+\left(u^{0} \nabla_{y}\right) \omega^{0}=0 \quad \text { in } Q_{T} \times \mathcal{Y}, \\
\omega^{0}(x, y, 0)=0 \quad \text { in } \Omega \times \mathcal{Y}, \quad \omega^{0} \text { is } 1 \text {-periodic in } y .
\end{array}\right.
$$

For the transport equation $\left(3.4^{A}\right), S$ is a characteristic boundary and we do not need any boundary condition on it. Therefore, $\omega^{0}=0$ and we conclude that smooth solutions for (2.7)-(2.10) have the fast scale vorticity equal to zero.

Step 2. Let us suppose that the system (2.7)-(2.10) has two different smooth solutions. Then their difference, which we denote by $\left\{v^{0}, \tilde{\pi}, \pi^{0}\right\}$, satisfies the homogeneous linear system

$$
\left\{\begin{array}{l}
\frac{\partial v^{0}}{\partial t}+\nabla_{x} \pi^{0}+\nabla_{y}\left(\tilde{\pi}+\frac{1}{2}\left(\left|u^{10}\right|^{2}-\left|u^{20}\right|^{2}\right)\right)=0 \text { in } Q_{T} \times \mathcal{Y}, \\
\operatorname{div}_{y}\left(v^{0}\right)=0 \text { in } Q_{T} \times \mathcal{Y}, \quad v^{0} . \nu=0 \text { in } Q_{T} \times S, \\
v^{0}(x, y, 0)=0 \text { in } \Omega \times \mathcal{Y}, \quad\left\{v^{0}, \tilde{\pi}\right\} \text { is } 1 \text {-periodic in } y \\
\operatorname{div}_{x}\left(\int_{\mathcal{Y}} v^{0}(x, y, t) d y\right)=0 \text { in } \Omega \times(0, T),\left\{v^{0}, \tilde{\pi}, \pi^{0}\right\} \text { is } L \text {-periodic in } x .
\end{array}\right.
$$

We note that using the Lamb formula and the result of step 1, the inertial terms $\left(u^{i 0} \nabla_{y}\right) u^{i 0}$ reduce to $\nabla_{y}\left(\frac{1}{2}\left|u^{i 0}\right|^{2}\right)$. Now the energy equality gives $v^{0}=0$ and $\left(3.5^{A}\right)$ reduces to:

$$
\nabla_{x} \pi^{0}+\nabla_{y} \tilde{\pi}=0 \quad \text { in } Q_{T} \times \mathcal{Y} .
$$

Thus, we obtain that $\nabla_{y} \tilde{\pi}$ is a constant with respect to $y$. Taking into account the periodicity in $y$, we obtain $\tilde{\pi}$ as a function of $(x, t)$ only. Using now the condition (2.18), we get $\int_{\mathcal{Y}} \tilde{\pi} d y=0$ and finally $\tilde{\pi}=0$. The same kind of argument gives also $\pi^{0}=0$ and the proposition is proved.

\section{Convergence of the homogenization process}

In this section, we justify rigorously the first terms in the asymptotic expansions (2.5) and (2.6), i.e. we prove that the differences $u^{\varepsilon}-u^{0}\left(x, \frac{x}{\varepsilon}, t\right)$ and $\varepsilon p^{\varepsilon}-p^{0}(x, t)$ are of order $\varepsilon$ in appropriate norms. 
Let $u^{0 \varepsilon}(x, t)=u^{0}\left(x, \frac{x}{\varepsilon}, t\right)$ and $p^{1 \varepsilon}=p^{1}\left(x, \frac{x}{\varepsilon}, t\right)$ for $(x, t) \in \Omega^{\varepsilon} \times(0, T)$, and $u^{0 \varepsilon}(x, t)=0$ for $(x, t) \in\left(\Omega \backslash \overline{\Omega^{\varepsilon}}\right) \times(0, T)$. Obviously $u^{0 \varepsilon}$ and $\partial_{t} u^{0 \varepsilon}$ are uniformly bounded in $L^{\infty}\left(0, T ; H\left(\Omega^{\varepsilon}, \operatorname{div}\right)\right)$, where

$$
\begin{aligned}
H\left(\Omega^{\varepsilon}, \operatorname{div}\right)=\left\{v \in L^{2}\left(\Omega^{\varepsilon}\right)^{2}: \operatorname{div}(v) \in L^{2}\left(\Omega^{\varepsilon}\right), v . \nu=0 \text { on } \partial \Omega^{\varepsilon} \backslash \partial \Omega\right. \\
\\
\text { and } v . \nu \text { is } \Omega \text {-antiperiodic }\} .
\end{aligned}
$$

However, $\operatorname{div}\left(u^{0 \varepsilon}\right)$ is not small and it introduces important compressibility effects. We follow W. Jäger, A. Mikelić [3] and introduce the following auxiliary problem:

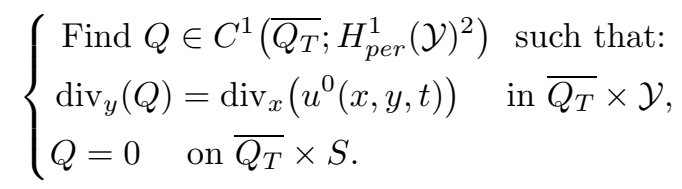

Owing to $(2.10),(4.1)$ has a solution $Q \in C^{1}\left(\overline{Q_{T}} ; C_{p e r}^{1}(\mathcal{Y})^{2}\right)$. Now we set $Q^{\varepsilon}(x, t)=$ $\varepsilon Q\left(x, \frac{x}{\varepsilon}, t\right)$ for $(x, t) \in \Omega^{\varepsilon} \times(0, T)$ and $Q^{\varepsilon}=0$ for $(x, t) \in\left(\Omega \backslash \overline{\Omega^{\varepsilon}}\right) \times(0, T)$.

The convergence result reads as follows:

Theorem 4.1. Suppose $f \in C_{\text {per }}^{\infty}\left(Q_{T}\right)^{2}$ and $u_{0} \in \mathcal{H}_{\text {per }}^{\infty}$ such that $\operatorname{curl}_{y}\left(u_{0}\right)=0$. Let $\left\{u^{\varepsilon}, p^{\varepsilon}\right\}$ be the solution for (1.2)-(1.5) and $\left\{u^{0}, p^{1}, p^{0}\right\}$ the solution for (2.7)(2.10) with (2.18). Then we have:

$$
\left\|u^{\varepsilon}-u^{0 \varepsilon}+Q^{\varepsilon}\right\|_{C^{1}\left([0, T] ; H_{p e r}(\Omega, \operatorname{div})\right)} \leq C \varepsilon
$$

Furthermore

$$
\sup _{0 \leq t \leq T}\left\|\left(\varepsilon p^{\varepsilon}-p^{0}\right)(t)\right\|_{L^{1}\left(\Omega^{\varepsilon}\right)} \leq C \varepsilon
$$

In order to prove theorem 4.1, we mimic the ideas from A. Mikelić [8] and find the equations satisfied by $u^{\varepsilon}-u^{0 \varepsilon}+Q^{\varepsilon}$. After subtracting the appropriate expressions, we get the following linear system for $\pi^{\varepsilon}=\varepsilon p^{\varepsilon}+\frac{\varepsilon}{2}\left|u^{\varepsilon}\right|^{2}-p^{0}-\varepsilon p^{1 \varepsilon}-\frac{\varepsilon}{2}\left|u^{0 \varepsilon}\right|^{2}$ and $w^{\varepsilon}=u^{\varepsilon}-u^{0 \varepsilon}+Q^{\varepsilon}$ :

$$
\left\{\begin{array}{l}
\frac{\partial w^{\varepsilon}}{\partial t}+\nabla \pi^{\varepsilon}=\psi^{\varepsilon} \quad \text { in } \Omega^{\varepsilon} \times(0, T) \\
\operatorname{div}\left(w^{\varepsilon}\right)=-\operatorname{div}_{x}\left(Q^{\varepsilon}\right) \quad \text { in } \Omega^{\varepsilon} \times(0, T), \quad w^{\varepsilon}(0)=0 \quad \text { in } \Omega^{\varepsilon} \\
w^{\varepsilon} \cdot \nu=0 \quad \text { on }\left(\partial \Omega^{\varepsilon} \backslash \partial \Omega\right) \times(0, T), \quad\left\{w^{\varepsilon}, \pi^{\varepsilon}\right\} \text { is } \Omega \text {-periodic }
\end{array}\right.
$$

where

$$
\psi^{\varepsilon}=\varepsilon u^{\varepsilon} \wedge \operatorname{curl}\left(u^{\varepsilon}\right)+\frac{\partial Q^{\varepsilon}}{\partial t}-\varepsilon\left(\nabla_{x}\left(p^{1 \varepsilon}\right)+\frac{1}{2} \nabla_{x}\left(\left|u^{0 \varepsilon}\right|^{2}\right)\right) .
$$

Using Proposition 2.1 and regularity properties of $u^{0}, p^{1}$ and $Q$ we obtain immediately the estimate

$$
\left\|\psi^{\varepsilon}\right\|_{L^{\infty}\left(0, T ; L^{2}\left(\Omega^{\varepsilon}\right)^{2}\right)} \leq C \varepsilon .
$$

It remains to estimate the pseudo-pressure term $\pi^{\varepsilon}$ using $\partial_{t} w^{\varepsilon}$ and then the multiplication by $\partial_{t} w^{\varepsilon}$ will give the required estimate for the "residual" velocity field $w^{\varepsilon}$. 
However, if the velocity is easily extended by zero to $\left(\Omega \backslash \overline{\Omega^{\varepsilon}}\right) \times(0, T)$ and its $H\left(\Omega^{\varepsilon}\right.$, div)-norm conserved, this is not the case with the pressure. In analogy with Tartar's construction in the homogenization of the Stokes flow through porous media, we need a restriction operator, which in our situation acts between

$$
H_{\text {per }}(\Omega, \operatorname{div})=\left\{v \in L^{2}(\Omega)^{2}: \operatorname{div}(v) \in L^{2}(\Omega) \text { and } v . \nu \text { is } \Omega \text {-antiperiodic }\right\},
$$

and $H\left(\Omega^{\varepsilon}\right.$, div $)$, and preserves the divergence-free vectors. We have:

Lemma 4.2. There exists a linear continuous operator

$$
R_{\varepsilon}: H_{p e r}(\Omega, \operatorname{div}) \rightarrow H\left(\Omega^{\varepsilon}, \operatorname{div}\right),
$$

such that:

$$
\begin{gathered}
\left\{\begin{array}{l}
R_{\varepsilon} v=v \quad \text { in } \Omega^{\varepsilon} \text { if } v \in H\left(\Omega^{\varepsilon}, \operatorname{div}\right), \\
\operatorname{div}\left(R_{\varepsilon} v\right)=0 \quad \text { in } \Omega^{\varepsilon} \text { if } \operatorname{div} v=0 \text { in } \Omega,
\end{array}\right. \\
\left\{\begin{array}{l}
\left\|R_{\varepsilon} v\right\|_{L^{2}\left(\Omega^{\varepsilon}\right)^{2}} \leq C\left(\|v\|_{L^{2}(\Omega)^{2}}+\varepsilon\|\operatorname{div} v\|_{L^{2}(\Omega)}\right), \\
\left\|\operatorname{div}\left(R_{\varepsilon} v\right)\right\|_{L^{2}\left(\Omega^{\varepsilon}\right)} \leq C\|\operatorname{div} v\|_{L^{2}(\Omega)},
\end{array}\right.
\end{gathered}
$$

for any $v \in H_{\text {per }}(\Omega$, div $)$.

Proof. Step 1. Let $v \in L^{2}(Y)^{2}$, $\operatorname{div} v \in L^{2}(Y)$. Then there exists a unique solution in $H(\mathcal{Y}$, div) denoted $R v$, of the following Neumann problem:

$$
\left\{\begin{array}{l}
\operatorname{div}(R v)=\operatorname{div} v+\frac{1}{|\mathcal{Y}|} \int_{\mathcal{O}} \operatorname{div} v \quad \text { in } \mathcal{Y}, \\
R v=\nabla \phi+v \quad \text { in } \mathcal{Y}, \\
R v . \nu=0 \quad \text { on } S, \quad R v . \nu=v . \nu \quad \text { on } \partial Y .
\end{array}\right.
$$

Standard estimates for a nonhomogeneous Neumann problem for Laplace's operator yield to:

$$
\begin{gathered}
\|R v\|_{L^{2}(\mathcal{Y})^{2}} \leq C\|v\|_{H(Y, \operatorname{div})}, \\
\|\operatorname{div}(R v)\|_{L^{2}(\mathcal{Y})} \leq C\|\operatorname{div} v\|_{L^{2}(Y)} .
\end{gathered}
$$

Step 2. Following the classical construction from homogenization of the Stokes flow through porous media, we rescale $R$ from $Y$ to $\varepsilon(Y+k)$ and obtain an operator $R_{\varepsilon}$ satisfying (4.6)-(4.7). For details we refer to L. Tartar [9] or G. Allaire [1].

Proposition 4.3. Let $w^{\varepsilon}$ and $\pi^{\varepsilon}$ be given smooth functions satisfying (4.4). Let $R_{\varepsilon}$ be the restriction operator defined by lemma 4.2 and $F^{\varepsilon}$ be given by

$$
\left\langle F^{\varepsilon}, \varphi\right\rangle_{\Omega}=\left\langle\nabla \pi^{\varepsilon}, R_{\varepsilon}(\varphi)\right\rangle_{\Omega^{\varepsilon}}, \quad \forall \varphi \in H_{p e r}(\Omega, \operatorname{div}) .
$$

Then there exists an unique pressure field $\tilde{\pi}^{\varepsilon}$ (the extension of $\pi^{\varepsilon}$ ) such that $F^{\varepsilon}=$ $\nabla \tilde{\pi}^{\varepsilon}$ in $\left(H_{\text {per }}(\Omega, \text { div })\right)^{\prime}, \int_{\Omega} \tilde{\pi}^{\varepsilon} d x=0$, for all $t \in[0, T]$. Furthermore, $\tilde{\pi}^{\varepsilon}=$ $\frac{1}{\varepsilon^{2}|\mathcal{Y}|} \int_{\varepsilon(\mathcal{Y}+k)} \pi^{\varepsilon}(x, t) d x$ in each solid part $\varepsilon(\mathcal{O}+k)$ and we have the following a priori estimate:

$$
\sup _{0 \leq t \leq T}\left\|\tilde{\pi}^{\varepsilon}(t)\right\|_{L_{0}^{2}(\Omega)} \leq C\left(\varepsilon+\left\|\partial_{t} w^{\varepsilon}\right\|_{L^{\infty}\left(0, T ; L^{2}\left(\Omega^{\varepsilon}\right)^{2}\right)}\right) .
$$


Proof. The first equation in (4.4) gives:

$$
\left\langle\nabla \pi^{\varepsilon}(t), \varphi\right\rangle_{\Omega^{\varepsilon}}=-\int_{\Omega^{\varepsilon}} \partial_{t} w^{\varepsilon}(t) \varphi+\int_{\Omega^{\varepsilon}} \psi^{\varepsilon}(t) \varphi
$$

for all $\varphi \in L^{2}\left(\Omega^{\varepsilon}\right)^{2}$. Owing to (4.5), we get

$$
\left|\left\langle\nabla \pi^{\varepsilon}(t), \varphi\right\rangle_{\Omega^{\varepsilon}}\right| \leq C\left(\varepsilon+\left\|\partial_{t} w^{\varepsilon}(t)\right\|_{L^{2}\left(\Omega^{\varepsilon}\right)^{2}}\right)\|\varphi\|_{L^{2}\left(\Omega^{\varepsilon}\right)^{2}}
$$

Therefore equations (4.7), (4.8) and (4.10) imply that $F^{\varepsilon}$ is uniformly bounded in $\left(H_{p e r}(\Omega, \operatorname{div})\right)^{\prime}$ for all $t$. Now we follow L. Tartar [9] and find out that (4.6) implies $F^{\varepsilon}=\nabla \tilde{\pi}^{\varepsilon}$. Since the divergence operator is surjective from $H_{p e r}(\Omega, \operatorname{div})$ to $L_{0}^{2}(\Omega)$, (4.7), (4.8) and (4.10) and a simple duality argument imply (4.9).

The explicit formula for the pressure extension is now obtained on the identical way as in the homogenization of the Stokes flow through porous media. We refer to G. Allaire [1] for details.

Now we have all necessary tools to complete the

Proof of Theorem 4.1. We multiply the first equation in (4.4) by $\partial_{t} w^{\varepsilon}(t)$ and integrate over $\Omega^{\varepsilon}$. It gives the equality

$$
\left\|\partial_{t} w^{\varepsilon}(t)\right\|_{L^{2}\left(\Omega^{\varepsilon}\right)^{2}}^{2}+\int_{\Omega^{\varepsilon}} \nabla \pi^{\varepsilon}(t) \partial_{t} w^{\varepsilon}(t)=\int_{\Omega^{\varepsilon}} \psi^{\varepsilon}(t) \partial_{t} w^{\varepsilon}(t),
$$

and consequently,

$$
\left\|\partial_{t} w^{\varepsilon}(t)\right\|_{L^{2}\left(\Omega^{\varepsilon}\right)^{2}}^{2} \leq\left|\int_{\Omega^{\varepsilon}} \pi^{\varepsilon}(t) \operatorname{div}\left(\partial_{t} w^{\varepsilon}(t)\right)\right|+\left|\int_{\Omega^{\varepsilon}} \psi^{\varepsilon}(t) \partial_{t} w^{\varepsilon}(t)\right| .
$$

Owing to the equality $\operatorname{div}\left(\partial_{t} w^{\varepsilon}\right)=\operatorname{div}_{x}\left(\partial_{t} Q^{\varepsilon}\right)$, definition of $Q^{\varepsilon}$ and the estimates (4.5) and (4.9), we obtain

$$
\left\|\partial_{t} w^{\varepsilon}(t)\right\|_{L^{2}\left(\Omega^{\varepsilon}\right)^{2}}^{2} \leq C \varepsilon\left(\varepsilon+\left\|\partial_{t} w^{\varepsilon}(t)\right\|_{L^{2}\left(\Omega^{\varepsilon}\right)^{2}}\right)+C \varepsilon\left\|\partial_{t} w^{\varepsilon}(t)\right\|_{L^{2}\left(\Omega^{\varepsilon}\right)^{2}},
$$

which proves (4.2). Then (4.2) and (4.9) imply

$$
\sup _{0 \leq t \leq T}\left\|\varepsilon p^{\varepsilon}+\frac{\varepsilon}{2}\left|u^{\varepsilon}\right|^{2}-p^{0}-\varepsilon p^{1 \varepsilon}-\frac{\varepsilon}{2}\left|u^{0 \varepsilon}\right|^{2}\right\|_{L^{1}\left(\Omega^{\varepsilon}\right)} \leq C \varepsilon,
$$

and (4.3) follows immediately.

Corollary 4.4. Let $\tilde{p}^{\varepsilon}$ be the extension of $p^{\varepsilon}$ defined by the analogue of (4.8). Then with the same notation as in Theorem 4.1 we have:

$$
\begin{gathered}
\varepsilon \tilde{p}^{\varepsilon} \rightarrow p^{0} \quad \text { in } C\left([0, T] ; L^{1}(\Omega)\right), \\
\partial_{t} u^{\varepsilon} \rightarrow \partial_{t} u_{E}=K_{E}\left(f-\nabla_{x} p^{0}\right) \quad \text { weak-* in } L^{\infty}\left(0, T ; L^{2}(\Omega)^{2}\right),
\end{gathered}
$$

as $\varepsilon \rightarrow 0$.

Remark 4.5. The volume $|\mathcal{Y}|$ of the fluid part $\mathcal{Y}$ of the unit cell $Y$ is called the porosity. If $\mathcal{O}$ is simply connected and $1-|\mathcal{Y}|=|\mathcal{O}|<<1$, then it is possible to determine accurately $K_{E}$ in an almost explicit way. More precisely, it was proved in V.V. Zhikov et al. [10] that:

$$
K_{E}=|\mathcal{Y}| I-M+o(1-|\mathcal{Y}|),
$$


where $M$ is the virtual mass tensor. In the case of a simple body $\mathcal{O}, M$ can be explicitely calculated. For example, if $\mathcal{O}$ is a ball, then $M=(1-|\mathcal{Y}|) I$ and, consequently,

$$
K_{E}=(2|\mathcal{Y}|-1) I+o(1-|\mathcal{Y}|) .
$$

For the details, we refer to V.V. Zhikov et al. [10].

\section{REFERENCES}

[1] Allaire G., One-phase newtonian flow, p. 45-68, in Homogenization and porous media, edited by U.Hornung, Springer, New York, 1997. CMP 97:08

[2] Bourgeat A., Marušić-Paloka E., Mikelić A., The weak non linear corrections for Darcy's law, Math. Models Methods Appl. Sci., 6(1996), 1143-1155. MR 98c:35126

[3] Jäger W., Mikelić A., On the boundary conditions at the contact interface between a porous medium and a free fluid, Ann. Scuola Norm. Sup. Pisa, Cl. Fisiche e Matematiche (4), 23(1996), 403-465. CMP 97:10

[4] Judovič V.I., A two dimensional problem of unsteady flow of an ideal incompressible fluid accross a given domain, Mat. Sb. (N.S) 31(1840), (1964), 562-588, and 64(106), (1964), 277304 (English translation). MR 31:1840

[5] Lions P.L., Mathematical topics in fluid mechanics, Vol 1: Incompressible models, Clarendon Press, Oxford, 1996. MR 98b:76001

[6] Marchioro C., Pulvirenti M., Mathematical theory of incompressible nonviscous fluids, Springer, New York, 1994. MR 94k:76001

[7] Marušić-Paloka E., Mikelić A., The derivation of a non-linear filtration law including the inertia effects via homogenization, Nonlinear Analysis, Theory, Methods and Applications, 1998, accepted for publication.

[8] Mikelić A., Effets inertiels pour un écoulement stationnaire visqueux incompressible dans un milieu poreux, C.R. Acad. Sci. Paris, Sér. I Math., 320(1995), 1289-1294. MR 96e:76124

[9] Tartar L., Convergence of the homogenization process, appendix to Lecture Notes in Physics 127, Springer, Berlin, 1980.

[10] Zhikov V.V., Kozlov S.M., Oleinik O.A., Homogenization of differential operators and integral functionals, Springer, New York, 1994. MR 96h:350036

Equipe D'Analyse Numérique Lyon-SAint Etienne, UMR 5585 CNRS, Université Lyon 1, 43 boulevard du 11 novembre 1918, 69622 Villeurbanne Cedex, France

E-mail address: andro@iris.univ-lyon1.fr

Equipe D'Analyse Numérique Lyon-Saint Etienne, UMR 5585 CNRS, Université de Saint Etienne, 23 rue du Docteur P.Michelon, 42023 Saint Etienne Cedex, France

E-mail address: paoli@anumsun1.univ-st-etienne.fr 